Regular Article

\title{
Shear band dilatation in amorphous alloys
}

\author{
M.Q. Jiang ${ }^{\mathrm{a}, \mathrm{b}, *}, \mathrm{G}$. Wilde ${ }^{\mathrm{c}}$, L.H. Dai ${ }^{\mathrm{a}, \mathrm{b}, *}$ \\ a State Key Laboratory of Nonlinear Mechanics, Institute of Mechanics, Chinese Academy of Sciences, Beijing 100190, China \\ b School of Engineering Science, University of Chinese Academy of Sciences, Beijing 100049, China \\ c Institute of Materials Physics, Westfälische Wilhelms-Universität Münster, Münster 48149, Germany
}

\section{A R T I C L E I N F O}

\section{Article history:}

Received 20 July 2016

Received in revised form 31 August 2016

Accepted 31 August 2016

Available online $\mathrm{xxxx}$

\section{Keywords:}

Amorphous alloys

Shear band

Dilatation

Free volume

Shear transformations

\begin{abstract}
A B S T R A C T
Based on the physical picture of free-volume creation and diffusion via shear transformations (STs), we develop a model for predicting the shear band dilatation in amorphous alloys. The model reveals that the dilatation in shear bands with fixed thickness increases with decreasing activation volume of STs that determines the diffusion distance of free-volume. Mature (or thicker) shear bands possess higher dilatation than recently evolved, thinner shear bands. The predicted dilatations agree well with the values of measurements and simulations, clarifying the long-standing controversy. The underlying physics is attributed to the local topological instability via STs that leads to shear banding.
\end{abstract}

(c) 2016 Acta Materialia Inc. Published by Elsevier Ltd. All rights reserved.
In 1885 , Osborne Reynolds pointed out that dilatancy is inherent to granular media that expand in response to shear [1]. It has been widely accepted that such shear-induced dilatation, as a mechanism for structural softening, can trigger the formation of shear bands in granular deformation [2] and flow [3]. Of great interest is that a similar picture can apply to the atomic scale. There is a general consensus that shear banding in amorphous alloys originates from the atomic-scale dilatation due to the shear of randomly close-packed atoms [4-9], although a secondary thermal softening is possibly involved [10-11]. Sustained efforts have been made to quantify the degree of dilatation or softening within amorphous shear bands [8,12-17]. However, the dilatation values determined by experiments and simulations showed a rather broad range from below $1 \%$ to more than $10 \%$. Naturally one would like to ask whether this dispersion is structure-inherent or not.

Recently, Shao et al. [18] have observed a high-resolution transmission electron microscopy (TEM) image of an amorphous shear band with two distinct "grey-white" zones. Fourier transformation revealed $4.5 \%$ and $11.8 \%$ volume dilatation, respectively, corresponding to the grey and white zones. They proposed that the former is contributed to co-shearing among atomic clusters, while the latter is due to internalshearing of individual clusters. This implies that the shear band dilatation closely relates to the underlying plastic events, although their quantitative relation has never been established. In this paper, we develop a model for predicting the shear band dilatation in amorphous

\footnotetext{
* Corresponding authors at: State Key Laboratory of Nonlinear Mechanics, Institute of Mechanics, Chinese Academy of Sciences, Beisihuanxi Road 15, Beijing 100190, China.

E-mail addresses: mqjiang@imech.ac.cn (M.Q. Jiang), lhdai@Inm.imech.ac.cn (L.H. Dai).
}

alloys within the physical picture of the free volume dynamics via shear transformations (STs) [9].

It is generally believed that amorphous plasticity is mediated by a cascade of "shear transformations" (STs), i.e., cooperative arrangements of local clusters of atoms [5-6,9]. Argon has pointed out that the net effect of such stress-driven STs is an excess dilatation that must create at least temporarily additional excess free-volume [5]. Competing with this excess volume increase, relaxation occurs in which the created excess free-volume diffuses via a series of STs but under the governance of thermal fluctuations [9]. The balance of excess free-volume creation and its relaxation determines a critical wavelength [11,19]:

$\lambda_{\text {crit }}=2 \pi \sqrt{\frac{D}{\partial G / \partial \xi}}$,

where Dand $G$ are, respectively, the diffusion coefficient and the net creation rate of free-volume $v_{f}$, and $\xi$ is the free-volume concentration that is defined as the free-volume $v_{f}$ normalized by the product of a geometrical factor $\chi$ and the effective hard-sphere size of an atom $v^{*}$. It is physically clear that $\xi$ directly measures the average volume expansion or dilatational strain during STs. Jiang and Dai [11] further revealed that a shear band will emerge in amorphous alloys if the wavelength of an external perturbation is larger than $\lambda_{\text {crit }}$; the characteristic thickness $T$ of the shear band is of the order of $\lambda_{\text {crit }}[20-21]$. Therefore, Eq. (1) provides a possible way to theoretically predict $\xi$ or the average dilatation within a shear band for a fixed thickness $T$.

Prior to prediction however, the free-volume creation and diffusional relaxation during shear banding needs to be described. It is 
reasonable to assume that shear banding occurs at the flow stress level $\tau_{y}$. By extending the free-volume concept towards so-called "flow defects", that are conceptually similar to the "STs", Spaepen and co-workers [22] derived the free-volume creation rate at the $\tau_{y}$ level:

$G=\frac{1}{\chi v^{*}} \frac{k_{B} \theta}{S} \frac{\Omega}{\Omega_{a}} \frac{1}{\xi} \dot{\gamma}^{p}$

where $k_{B} \theta$ is the energy of thermal fluctuations, $S=2(1+\nu) \mu / 3(1-\nu)$ is the Eshelby modulus with Poisson's ratio $\nu$ and the shear modulus $\mu$, $\Omega$ is the average atomic volume, $\Omega_{a}$ is the activation volume of microscopic plastic events (here "STs" or "flow defect"), and $\dot{\gamma}^{p}$ is the plastic strain rate. On the other hand, the diffusion coefficient of the freevolume is assumed to obey a modified Stokes-Einstein relation:

$D=\frac{k_{B} \theta}{6\left(v_{f} / d^{2}\right) \eta}$.

It should be noted that the characteristic diffusion distance $d$ of freevolume is not related to the average atomic size, but to the characteristic size of an ST event. This consideration coincides with the picture that the free-volume diffuses by means of collective motions of atoms, instead of single-atom jumps [23-25]. The shear viscosity can be determined as: $\eta=\tau_{y} / \dot{\gamma}^{p}$. Inserting the first derivative of Eq. (2) with respect to $\xi$ and Eq. (3) into Eq. (1), we can solve $\xi$ with the explicit expression:

$\xi=\frac{1}{2 \Gamma_{1}^{2} \Gamma_{2}}\left(T \sqrt{T^{2}+4 \Gamma_{1}^{2} \Gamma_{2}}-T^{2}\right)$,

where $\Gamma_{1}=\frac{2 \pi}{3} d$, and $\Gamma_{2}=\frac{1}{\gamma_{c}} \frac{(1+\nu)}{(1-\nu)} \frac{\Omega_{a}}{\Omega}$ with the critical shear yield strain $\gamma_{c}$ that is almost a universal constant of about 0.0267 for amorphous alloys at room temperature [26]. This Eq. (4) demonstrates that factors affecting the shear band dilatation $\xi$ are rather complex, at least including the diffusion distance $d$ of free-volume, Poisson's ratio $\nu$ and the activation volume $\Omega_{a} / \Omega$ of STs for a fixed thickness $T$. These parameters must be reasonably estimated for the further calculation.

As mentioned above, the diffusion distance $d$ of free-volume is assumed to be the characteristic size of an ST event. Based on the Johnson-Samwer cooperative shearing model [26], Pan et al. have developed a rate-jump nanoindentation method to estimate the size of the STs in amorphous alloys [27]. Their analysis revealed that the equivalent diameters of STs are about 1.3-1.9 nm for various systems. Mayr and co-workers have determined an STs' size of $0.8 \mathrm{~nm}-1.5 \mathrm{~nm}$ from molecular dynamics simulations [28-29]. A similar range from $0.85 \mathrm{~nm}$ (brittle systems) to $1.5 \mathrm{~nm}$ (ductile systems) was also obtained by Murali et al. [30]. Therefore, in the following calculation, we adopt that $d$ ranges from 0.8 to $2 \mathrm{~nm}$, with a characteristic value of about $1 \mathrm{~nm}$. According to the random close-packing of atoms, the STs' volume commonly includes about 100-1000 atoms. Considering the characteristic shear strain of about $\sim 0.1$ of a ST [31], the activation volume $\Omega_{a}$ of STs is obtained as $10-100 \Omega$, that is, $\Omega_{a} / \Omega \approx 10-100$. In addition, amorphous alloys usually have a Poisson's ratio $\nu$ of $\sim 0.28-0.42$ [32]. In general, the thickness $T$ of shear bands in amorphous alloys is restricted to a rather narrow range from several to 10 or more nanometers [33], regardless of systems and loading conditions; this has been widely confirmed by experiments [12,34-36], simulations [30,37-38] or theoretical prediction [21]. Recent experimental observations including correlative analytical transmission electron microscopy indicate the presence of two types of "shear bands" in amorphous alloys: thin shear bands that do not span the entire sample dimension with typical widths of about 4-7 nm and more macroscopic "shear bands" that resemble partially open cracks with typical widths exceeding $10 \mathrm{~nm}$ [17]. We thus choose $T=1-20 \mathrm{~nm}$ with a typical value of $10 \mathrm{~nm}$, thus representing those shear bands that contribute the major part of the macroscopic slip.

As a first step, we examined the effects of the diffusion distance $d$ of the excess free-volume and of Poisson's ratio $\nu$ on the shear band dilatation $\xi$ for fixed $\Omega_{a} / \Omega=100$ and $T=10 \mathrm{~nm}$, as shown in Fig. 1. The predicted dilatation $\xi$ varies from about $2.4 \%$ to $7.0 \%$, falling in the range reported from experiments [15,17]. Importantly, the results clearly indicate that the shear band dilatation increases with decreasing $d$ and/or $\nu$, but the effect of $\nu$ on $\xi$ is much less pronounced than the effect of $d$. This implies that the diffusion distance of free-volume is a governing factor; a shear band will possess higher dilatation if the diffusion of free-volume within it is slower. In fact, a short diffusion distance $d$ of free-volume corresponds to STs with a smaller activation volume $\Omega_{a} / \Omega$. We then explore the effect of the activation volume $\Omega_{a} / \Omega$ on the dilatation $\xi$ in shear bands with different thickness T. Fig. 2 shows the result for a typical Poisson's ratio $\nu=0.36$. As expected, the dilatation increases with decreasing activation volume for a fixed thickness. If the capability of STs to dilate, $\xi$, is similar, a larger activation volume or longer diffusion distance determine a thicker shear band. Therefore, the predicted dilatation usually ranges from corner " $\mathrm{A}$ " to corner "B" (Fig. 2), between $1.68 \%$ to $10.15 \%$. The dilatation data reported in the literature $[8,12-17]$ fall exactly in this range of predicted values. It is worth noting that the upper limit predicted here is very close to the critical volume expansion $(\sim 11 \%)$ above which liquid-like regions or $n$-type defects can be defined [13]. The present model also predicts that mature shear bands, characterized by a larger thickness, possess higher dilatation than more recently formed shear bands. Yet it should be noted that the thicker shear bands, i.e. larger activation volume or longer diffusion distance, in turn decrease the dilatation. Therefore, an optimum thickness of a shear band is determined by the balance between dilatation and

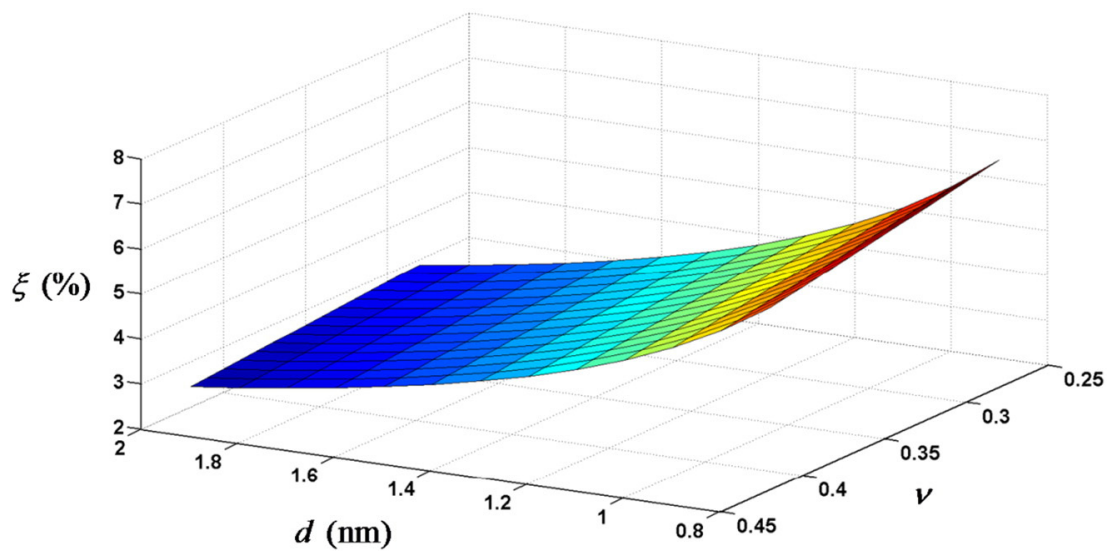

Fig. 1. Prediction of shear band dilatation $\xi$ with the changes of diffusion distance $d$ of free-volume and Poisson's ratio $\nu$. 


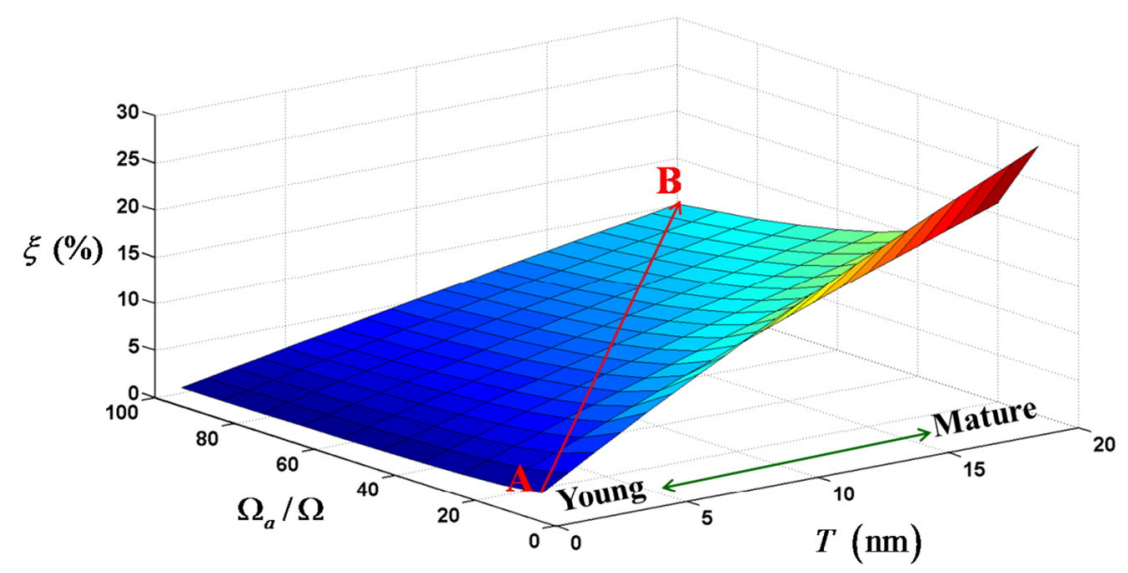

Fig. 2. Prediction of shear band dilatation $\xi$ with the change of activation volume $\Omega_{a} / \Omega$ of STs for different shear band thicknesses $T$.

diffusion, both by means of a series of STs, which was revealed previously [21].

The rationality of the theoretical prediction can be further supported by comparison with related work. The experiments of Shao et al. [18] revealed that the shear band dilatation $(\sim 11.8 \%)$ due to individual STs is larger than that $(\sim 4.5 \%)$ due to cooperative STs. Obviously, the former event corresponds to a smaller activation volume, and the latter to a larger activation volume. Our model (Fig. 2) indeed predicts that for a $10 \mathrm{~nm}$-thick shear band (close to that observed by Shao et al. [18]), the larger activation volume $\left(\Omega_{a} / \Omega=100\right)$ results in a lower dilatation of about $5.21 \%$, while the smaller $\left(\Omega_{a} / \Omega=20\right)$ leads to a higher dilatation of about $11.28 \%$. Murali et al. [30] have performed molecular dynamics simulations to examine how the ST size and shear band thickness are interrelated in amorphous alloys. They found that brittle systems exhibit smaller ST size (measured by a characteristic length scale), thinner shear bands and lower Poisson's ratios as compared with ductile systems. Our prediction agrees well with their results, if one unitedly looks at Figs. 1 and 2. More importantly, the present model can clarify the confusion why the shear band dilatation probed by Klaumünzer et al. [15] is below those reported in any earlier study $[12,39]$. We note that the dilatation values obtained by Klaumünzer et al. are associated with the shear band initiation or the "young" shear bands. However, earlier studies usually performed ex-situ measurements on mature shear bands. According to our analysis (Fig. 2), the "young" shear bands exhibit smaller dilatation than the mature shear bands. The underlying mechanism can be explained by the picture proposed by Shao et al. [18]. At the initial stage of deformation, cooperative rearrangements among atomic clusters take place [18], corresponding to the STs with relatively large activation volume. This kind of STs guarantees that the dilatation or free-volume created can diffuse over a long distance to widen the shear band. As deformation further develops, these individual clusters per se become unstable to form STs with relatively smaller activation volume. This in turn limits the diffusion of free volume and keeps the dilatation at a relatively high level.

Although shear bands in amorphous alloys are dilatant as a whole, local sites within them can be expanded or compacted. This was confirmed recently by Schmidt et al. [17] who explored local density changes in an amorphous shear band from the high-angle annular dark-field scanning TEM signal. They found that the shear band shows a spectrum of densities ranging from a slight increase to a significant decrease with an average decrease compared to the matrix of about $2.9 \%$. It has been proposed that the alternating changes in density, corresponding to a stick-slip motion of the shear band propagation, originate from the shear response to the nanoscale heterogeneity of amorphous alloys. The local expanded and compact sites agree well with the liquid (freevolume, $n$-type defects) and solid (anti-free-volume, $p$-type defects) regions defined by Egami according to the concept of atomic level stresses [13]. Our model captures the overall dilatancy of amorphous shear bands, but neglects the local fluctuations. In addition to the topological disorder, the chemical disorder arising from compositional changes may also contribute to the shear band dilatation, although the latter is not dominant [17].

In conclusion, we derive an explicit expression for the shear band dilatation in amorphous alloys by taking into account the free-volume creation and diffusion via STs. This model demonstrates that the dilatation within shear bands depends closely on the activation volume of STs that governs the diffusion distance of free-volume. The developing degree of a shear band, denoted by its thickness, also affects the resulting dilatation within it. Mature shear bands usually possess higher dilatation than the recently formed shear bands. The predicted dilatations agree well with the values of existing measurements and simulations. Our findings substantiate the idea that shear banding in amorphous alloys results from local topological instabilities of STs [18,21,30,33].

\section{Acknowledgements}

This work was supported by the National Nature Science Foundation of China (Grant Nos. 11522221, 11372315 and 11472287). GW acknowledges support by Deutsche Forschungsgemeinschaft.

\section{References}

[1] O. Reynolds, Philos. Mag. 20 (1885) 469.

[2] C.M. Gourlay, A.K. Dahle, Nature 445 (2007) 70.

[3] D.M. Mueth, G.F. Debregeas, G.S. Karczmar, P.J. Eng, S.R. Nagel, H.M. Jaeger, Nature 406 (2000) 385.

[4] F. Spaepen, Acta Metall. 25 (1977) 407

[5] A.S. Argon, Acta Metall. 27 (1979) 47.

[6] M.L. Falk, J.S. Langer, Phys. Rev. E 57 (1998) 7192

[7] A. Lemaître, Phys. Rev. Lett. 89 (2002) 195503.

[8] L. Li, E.R. Homer, C.A. Schuh, Acta Mater. 61 (2013) 3347.

[9] M.Q. Jiang, G. Wilde, L.H. Dai, Mech. Mater. 81 (2015) 72.

[10] J.J. Lewandowski, A.L. Greer, Nat. Mater. 5 (2006) 15.

[11] M.Q. Jiang, L.H. Dai, J. Mech. Phys. Solids 57 (2009) 1267

[12] P. Donovan, W.M. Stobbs, Acta Metall. 29 (1981) 1419.

[13] T. Egami, Prog. Mater. Sci. 56 (2011) 637.

[14] J. Pan, Q. Chen, L. Liu, Y. Li, Acta Mater. 59 (2011) 5146.

[15] D. Klaumünzer, A. Lazarev, R. Maaß, F.H. Dalla Torre, A. Vinogradov, J.F. Löffler, Phys. Rev. Lett. 107 (2011) 185502.

[16] Y.J. Wang, M.Q. Jiang, Z.L. Tian, L.H. Dai, Scr. Mater. 112 (2016) 37.

[17] V. Schmidt, H. Rösner, M. Peterlechner, G. Wilde, P.M. Voyles, Phys. Rev. Lett. 115 (2015) 035501

[18] Y. Shao, K. Yao, M. Li, X. Liu, Appl. Phys. Lett. 103 (2013) 171901.

[19] R. Huang, Z. Suo, J.H. Prevost, W.D. Nix, J. Mech. Phys. Solids 50 (2002) 1011.

[20] L.H. Dai, Y.L. Bai, Int. J. Impact Eng. 35 (2008) 704.

[21] M.Q. Jiang, W.H. Wang, L.H. Dai, Scr. Mater. 60 (2009) 1004.

[22] M. Heggen, F. Spaepen, M. Feuerbacher, J. Appl. Phys. 97 (2005) 033506.

[23] F. Faupel, P.W. Huppe, K. Rätzke, Phys. Rev. Lett. 65 (1990) 1219.

[24] X.-P. Tang, U. Geyer, R. Busch, W.L. Johnson, Y. Wu, Nature 402 (1999) 160

[25] V. Zollmer, K. Rätzke, F. Faupel, A. Rehmet, U. Geyer, Phys. Rev. B 65 (2002) 220201

[26] W.L. Johnson, K. Samwer, Phys. Rev. Lett. 95 (2005) 195501.

[27] D. Pan, A. Inoue, T. Sakurai, M.W. Chen, PNAS 105 (2008) 14769.

[28] S.G. Mayr, Phys. Rev. Lett. 97 (2006) 195501. 
[29] M. Zink, K. Samwer, W.L. Johnson, S.G. Mayr, Phys. Rev. B 73 (2006) 172203.

[30] P. Murali, Y.W. Zhang, H.J. Gao, Appl. Phys. Lett. 100 (2012) 201901.

31] C.A. Schuh, T.C. Hufnagel, U. Ramamurty, Acta Mater. 55 (2007) 4067.

[32] M.Q. Jiang, L.H. Dai, Phys. Rev. B 76 (2007) 054204.

[33] Y. Zhang, A.L. Greer, Appl. Phys. Lett. 89 (2006) 071907.

[34] T. Masumoto, R. Maddin, Acta Metall. 19 (1971) 725.
[35] J. Li, F. Spaepen, T.C. Hufnagel, Philos. Mag. 82 (2002) 2623.

[36] E. Pekarskaya, C.P. Kim, W.L. Johnson, J. Mater. Res. 16 (2001) 2513.

[37] Y.F. Shi, M.L. Falk, Phys. Rev. Lett. 95 (2005) 095502.

[38] A.J. Cao, Y.Q. Cheng, E. Ma, Acta Mater. 57 (2009) 5146-5155.

[39] A.S. Argon, J. Megusar, N.J. Grant, Scr. Metall. 19 (1985) 591. 